\title{
Effects of gravitational stresses on mitral valve prolapse I: Changes in auscultatory findings produced by progressive passive head-up tilt
}

\author{
WILLIAM D. TOWNE, RAMESH PATEL, JAIRO CRUZ, NEIL KRAMER, AND \\ KAMAL K. CHAWLA \\ From the Division of Adult Cardiology, Cook County Hospital, Chicago, Illinois, U.S.A.
}

SUMMARY The effects of passive head-up tilt on systolic time intervals were assessed in 18 patients with mitral valve prolapse. In addition to causing prolongation of the pre-ejection period and shortening of left ventricular ejection time, this circulatory stress led to progressive shortening of the $Q$ to click interval. In 1 patient, a systolic click became audible which had not been heard in the supine posture. In 7 patients the click disappeared during head-up tilt, usually at $60^{\circ}$ or $90^{\circ}$. In 2 patients without a murmur while supine, a mid-late systolic murmur appeared with tilt; 1 of these 2 as well as another patient who had a soft late systolic murmur while supine developed loud systolic whoops at greater angles of tilt. The correlations between $Q$ to click interval and aortic valve opening to click interval, and both the angle and the sine of the angle of tilt were highly significant.

During the past 15 years, it has been shown that the mid or late systolic click is most commonly associated with mitral valve prolapse (Barlow et al., 1968; Jeresaty, 1973). Mitral valve prolapse can be demonstrated by left ventriculography (Criley $e t$ al., 1966; Ranganathan et al., 1973) or echocardiography (Dillon et al., 1971; Kerber et al., 1971; Popp et al., 1974) and is often associated with mitral regurgitation which may produce either a holosystolic or, more commonly, a late systolic murmur.

Previous studies have shown that most interventions which diminish left ventricular volume move the click to an earlier position in systole (Fontana et al., 1975; Winkle et al., 1975). A late systolic murmur, if present, is usually lengthened by the same interventions. Fontana has shown that $45^{\circ}$ head-up tilt causes a reduction in left ventricular volume in patients with mitral valve prolapse with the expected effects on auscultatory findings. However, no previous study has been designed to test systematically the effect of progressive head-up tilt from the supine position to $90^{\circ}$ on the timing and characteristics of the systolic click and murmur.

Received for publication 13 October 1976

\section{Subjects and methods}

Eighteen patients with mitral valve prolapse who had mid or late systolic clicks constantly or intermittently audible and recordable while in the supine position formed the basis of this study. Three of the patients were men and 15 were women, and their average age was 32 years, with a range of 17 to 61 . None of the patients gave a history suggestive of rheumatic fever or coronary artery disease and none showed clinical evidence of left ventricular failure. One patient had a late systolic murmur while supine. The diagnosis of mitral valve prolapse was proven in 5 of the patients by left ventriculography. The remaining 13 patients were not subjected to invasive procedures.

All recordings were made with a HewlettPackard Model 564 polytrace recorder. All patients had recordings made consecutively at $0,15,0,30$, $0,45,0,60,0$, and 90 degrees of head-up tilt, after a 5-minute period of stabilisation in each position. The surface electrocardiogram, phonocardiograms (200 $\mathrm{Hz}$ and $24 \mathrm{Db} /$ octave) from the third left interspace parasternally and the apex, and an indirect carotid tracing were simultaneously recorded. The arterial blood pressure was measured by sphygo482 
manometer just before each recording.

The following time intervals were measured to the nearest $5 \mathrm{~ms}$ at $100 \mathrm{~mm} / \mathrm{s}$ paper speed and averaged over 5 to 10 cycles: cycle length (CL); total tlectromechanical systole (Q-S2); left ventricular ejection time (LVET); and time between the onset of electromechanical systole and the initial vibrations of the systolic click (Q-C). The following time intervals were derived from these: pre-ejection period (PEP); interval between the systolic click and the conclusion of the electromechanical systole (C-S2); and interval between the onset of ejection and the click (E-C). The pre-ejection period (PEP) was obtained by subtracting LVET from Q-S2. The interval between the click and S2 (C-S2) was derived by subtracting Q-C from Q-S2, and the interval between onset of left ventricular ejection and the click (E-C) was obtained by subtracting C-S2 from LVET. In one patient (case 10 in Table 2) recordings of the second sound were unsatisfactory in some tracings; Q-S2, C-S2, and E-C were not measured in this patient.

\section{Results}

Ten patients had a click heard and recorded at all angles of tilt as well as in the supine position (Fig. 1). In 8 patients the click was absent at one or more stages of the procedure. One patient in whom a late systolic click had been heard previously had no click audible or recordable in the supine position or at any angle of head-up tilt on the day of the test. The click was present intermittently and transiently at $+15^{\circ}$ before the 5-minutc stabilising period had elapsed and was brought out by $20^{\circ}$ of head-down tilt after the test was completed. When present, the click was loud and late systolic in timing. A classic echocardiographic pattern of mitral valve prolapse was shown in this patient.
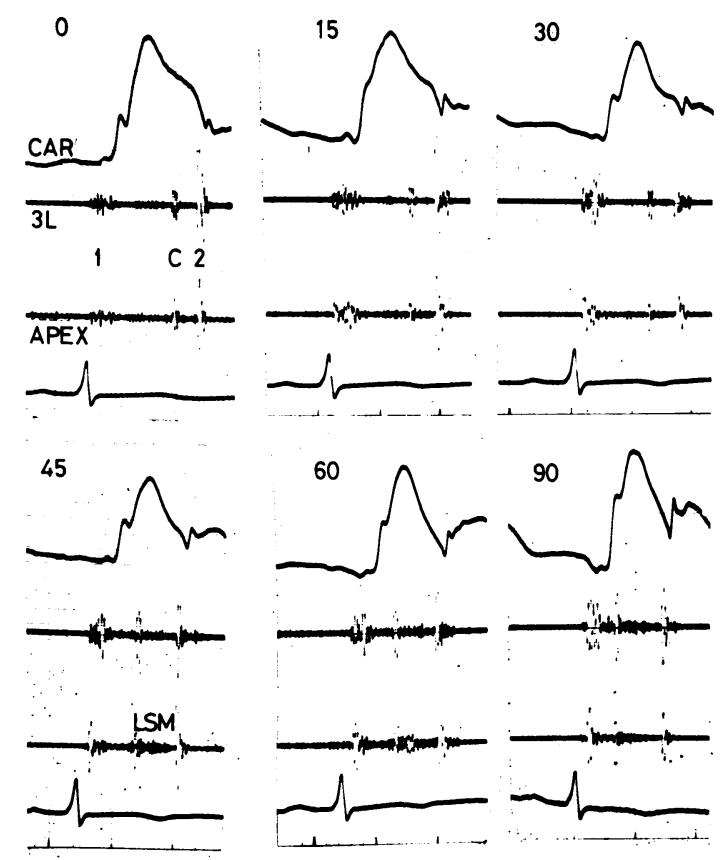

Fig. 1 A representative phonocardiogram from a patient recorded at $0^{\circ}, 15^{\circ}, 30^{\circ}, 45^{\circ}, 60^{\circ}$, and $90^{\circ}$ of head-up tilt. The click progressively moves towards $S 1$ and a murmur appears at $45^{\circ}$ tilt. 1 , first heart sound; 2 , second heart sound; C, click; LSM, late systolic murmur; CAR, indirect carotid tracing; $3 L$, third intercostal space left sternal border.

Another patient had no click audible or recordable while supine or at $15^{\circ}$ angle of tilt on the day of the test. However, the click was audible and recorded at all of the greater angles of tilt. In a third patient the click was absent at $30^{\circ}, 60^{\circ}$, and $90^{\circ}$ head-up tilt though present at $0^{\circ}, 15^{\circ}$, and $45^{\circ}$. In another subject the click was present at $0^{\circ}, 15^{\circ}$,

Table 1 Effects of head-up tilt on systolic time intervals

\begin{tabular}{|c|c|c|c|c|c|c|}
\hline Angle of tilt & $\begin{array}{l}C L \\
(m s \pm S E)\end{array}$ & $\frac{Q-S 2}{(m s \pm S E)}$ & $\begin{array}{l}L V E T \\
(m s \pm S E)\end{array}$ & $\underset{(m s \pm S E)}{Q-C}$ & $\begin{array}{l}E-C \\
(m s \pm S E)\end{array}$ & $\begin{array}{l}\text { Number of } \\
\text { patients } \\
\text { with click }\end{array}$ \\
\hline $\begin{array}{l}\text { Supine } \\
15^{\circ} \\
30^{\circ} \\
45^{\circ} \\
60^{\circ} \\
90^{\circ} \\
\text { Correlation coefficient (r) with: } \\
\text { sine of angle } \\
\text { angle }\end{array}$ & $\begin{array}{l}899 \pm 32 \\
857 \pm 37 \\
829 \pm 37 \\
756 \pm 37 \\
706 \pm 32 \\
669 \pm 36 \\
-0.9819 \\
-0.9806\end{array}$ & $\begin{array}{l}398 \pm 8 \\
383 \pm 8 \\
371 \pm 9 \\
354 \pm 9 \\
341 \pm 5 \\
324 \pm 11 \\
-0.9882 \\
-0.9928\end{array}$ & $\begin{array}{l}296 \pm 7 \\
279 \pm 8 \\
260 \pm 7 \\
243 \pm 7 \\
230 \pm 5 \\
222 \pm 7 \\
-0.9992 \\
-0.9652\end{array}$ & $\begin{array}{l}290 \pm 15 \\
272 \pm 17 \\
263 \pm 15 \\
224 \pm 15 \\
203 \pm 16 \\
184 \pm 19 \\
-0.9732 \\
-0.9777\end{array}$ & $\begin{array}{r}188 \pm 14 \\
168 \pm 17 \\
152 \pm 15 \\
113 \pm 14 \\
92 \pm 14 \\
82 \pm 17 \\
-0.9864 \\
-0.9431\end{array}$ & $\begin{array}{l}16 \\
16 \\
15 \\
15 \\
13 \\
12\end{array}$ \\
\hline
\end{tabular}

Data represent mean value \pm standard error of the mean. Data for the supine position represents the average of the five control periods. The values for Q-S2, LVET, and Q-C are uncorrected for heart rate.

The progressive changes in the intervals are highly significant in relation to sine of the angle of tilt $(P<0.001)$.

Abbreviations: CL, cycle length; Q-S2, total electromechanical systole; LVET, left ventricular ejection time; $Q-C$, interval between onset of QRS and click. 


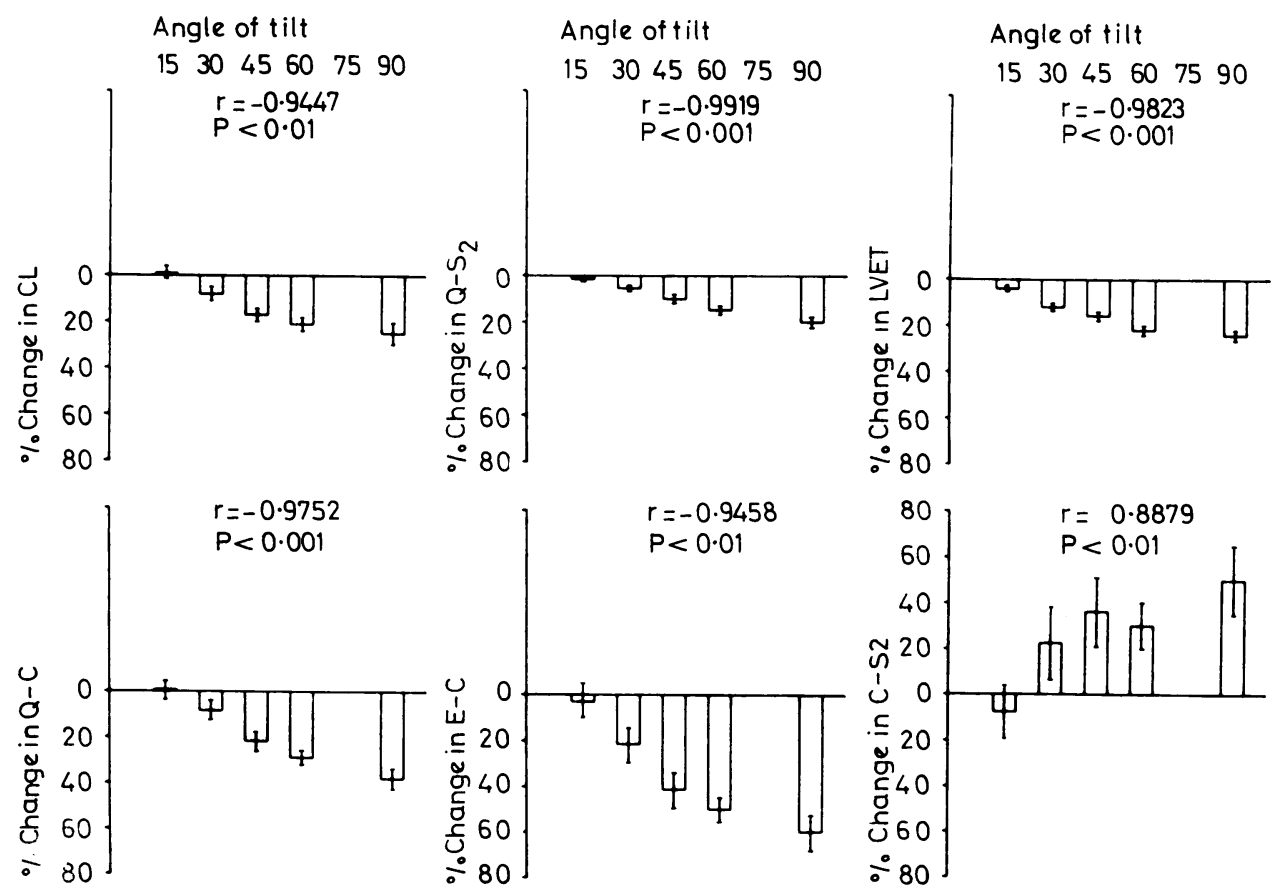

Fig. 2 Average percentage change in systolic time intervals in 10 patients with click recordable at all angles of tilt. The values are correlated with angle of tilt. The bracket represents standard error. Abbreviations: $C L$, cycle length; $Q-S 2$, electromechanical systole; $L V E T$, left ventricular ejection time; $Q-C$, onset of $Q R S$ to click interval; $E-C$, interval from beginning of LVET to click. C-S2, interval from click to second heart sound.

and $90^{\circ}$ but absent at $30^{\circ}, 45^{\circ}$, and $60^{\circ}$. In a fifth patient the click was absent at $45^{\circ}, 60^{\circ}$ and $90^{\circ}$ of head-up tilt. In a sixth subject the click was absent at $60^{\circ}$ angle of tilt but present at all other angles. In the 2 remaining patients the click was present at all angles except $90^{\circ}$.

A progressive decrease in Q-S2, LVET, Q-C, and E-C was observed as the angle of tilt was increased (Table 1). At the time of the recording, no patient had a significant change in blood pressure from the supine level at any angle of tilt. The correlation between $\mathrm{Q}-\mathrm{C}$ and the sine of the angle of tilt was highly significant $(r=-0.9732 ; P<$ 0.001 ), as was the correlation between $\mathrm{Q}-\mathrm{C}$ and the angle itself $(r=-0.9777 ; P<0.001)$.

The percentage changes in the measured and derived intervals with tilt from the immediately preceding control period are shown for the 10 patients in whom a click was audible at all angles of tilt in Figs. 2 and 3. The percentage change in Q-C correlated slightly better with the sine of the angle of tilt $(r=-0.9964)$ than with the angle itself $(r=-0.9752)$. However, both correlations were highly significant $(P<0.001)$. The correlation coefficients between percentage change in Q-C and $\mathrm{E}-\mathrm{C}$ and the angle and sine of the angle of tilt are shown in Table 2.

Fifteen patients had no mitral regurgitant systolic murmur while supine or at any angle of tilt. In one patient who had a mid-late systolic murmur preceded by a click while supine, the later portion of this murmur became musical with a whooping character at all angles of tilt above the horizontal (Fig. 4). A second patient who had no murmur while supine or at $15^{\circ}$ developed a soft midsystolic murmur at $30^{\circ}$ which became much louder and musical at $45^{\circ}$ and remained so at $60^{\circ}$ and $90^{\circ}$ (Fig. 5). This murmur was audible at a distance from the chest without a stethescope. In a third patient a mid-late systolic murmur appeared after the click at $45^{\circ}$ angle of tilt (Fig. 1).

\section{Discussion}

The timing of the systolic click in patients with mitral valve prolapse appears to be governed mainly by volume of the left ventricular cavity. As a result of a study on the effects of atrial and ventricular 
Sine of angle of tilt $0.2 \quad 0.4 \quad 0.60 .81 .0$

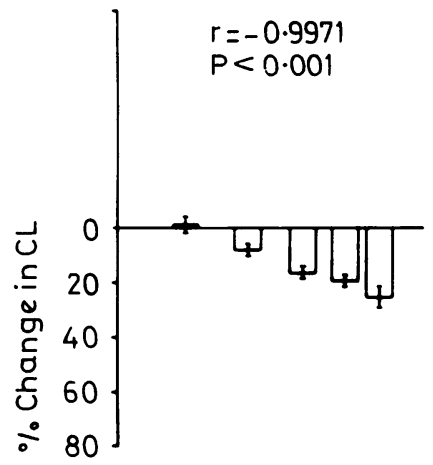

Sine of angle of tilt

$0.2 \quad 0.40 .60 .81 .0$

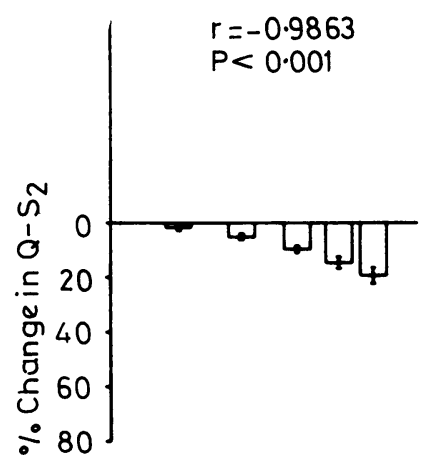

Sine of angle of tilt

$0.2 \quad 0.4 \quad 060.81 .0$
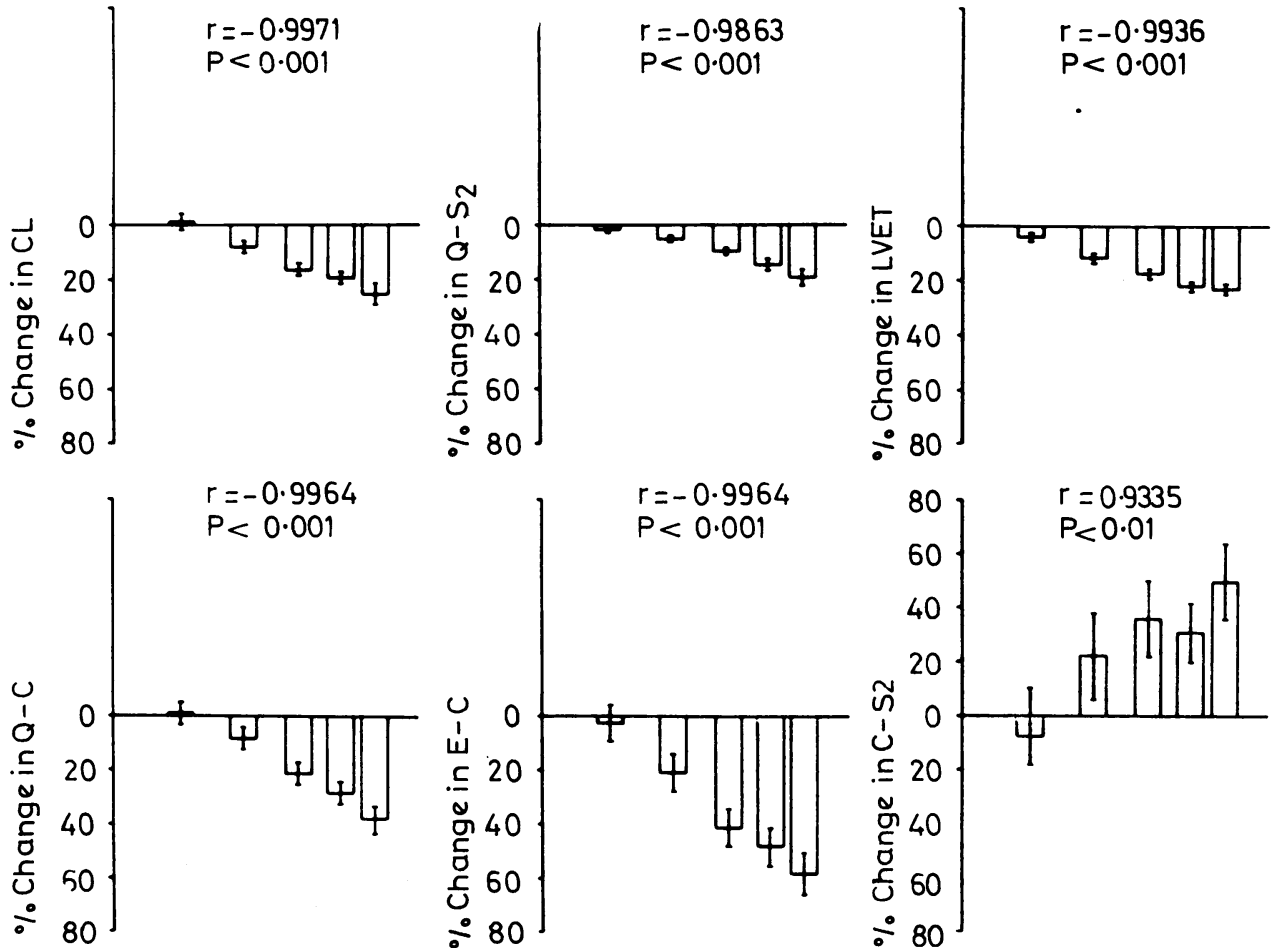

Fig. 3 Average percentage change in systolic time intervals in 10 patients with click recordable at all angles of tilt. The values are correlated with sine of angle of tilt. Bracket represents standard error. Abbreviations as in Fig. 2.

pacing on click position (Towne et al., 1975), we postulated that there was a critical left ventricular volume at which prolapse occurs for each patient with this condition. If this were the case, enddiastolic volume, stroke volume, and ejection rate would be the primary determinants of the presence and timing of a systolic click, and other circulatory factors including preload, heart rate, contractility, and systemic impedance, would be related indirectly to click presence and timing. Support for the 'critical prolapsing volume' (CPV) concept is provided by the echocardiographic work of Mathey et al. (1976). In view of the fact that the systolic time intervals (STI) in normal subjects are also influenced by multiple cardiovascular variables, yet show a predictable and linear response to the sine

Table 2 Correlation coefficients between percentage change in $Q-C$ and $E-C$ and angle of tilt in 10 patients with click recordable at all angles

\begin{tabular}{|c|c|c|c|c|}
\hline Case No. & $Q-C$ vs sine of angle & $Q-C$ vs angle & $E-C$ vs sine of angle & $E-C$ vs angle \\
\hline $\begin{array}{r}1 \\
2 \\
3 \\
4 \\
5 \\
6 \\
7 \\
8 \\
9 \\
10\end{array}$ & $\begin{array}{l}-0.7261 \\
-0.9425 \\
-0.9065 \\
-0.9535 \\
-0.9745 \\
-0.9155 \\
-0.9722 \\
-0.9388 \\
-0.9589 \\
-0.9686\end{array}$ & $\begin{array}{l}-0.5242 \\
-0.9954 \\
-0.7870 \\
-0.8547 \\
-0.9842 \\
-0.8345 \\
-0.9742 \\
-0.9919 \\
-0.9809 \\
-0.9103\end{array}$ & $\begin{array}{l}-0.7134 \\
-0.9844 \\
-0.9610 \\
-0.9449 \\
-0.9867 \\
-0.7392 \\
-0.9734 \\
-0.9409 \\
-0.9816 \\
*\end{array}$ & $\begin{array}{l}-0.5198 \\
-0.9486 \\
-0.8615 \\
-0.8594 \\
-0.9549 \\
-0.6528 \\
-0.9634 \\
-0.8669 \\
-0.9862 \\
*\end{array}$ \\
\hline
\end{tabular}

* Poor recording of the second sound at some angles prevented derivation of E-C. 


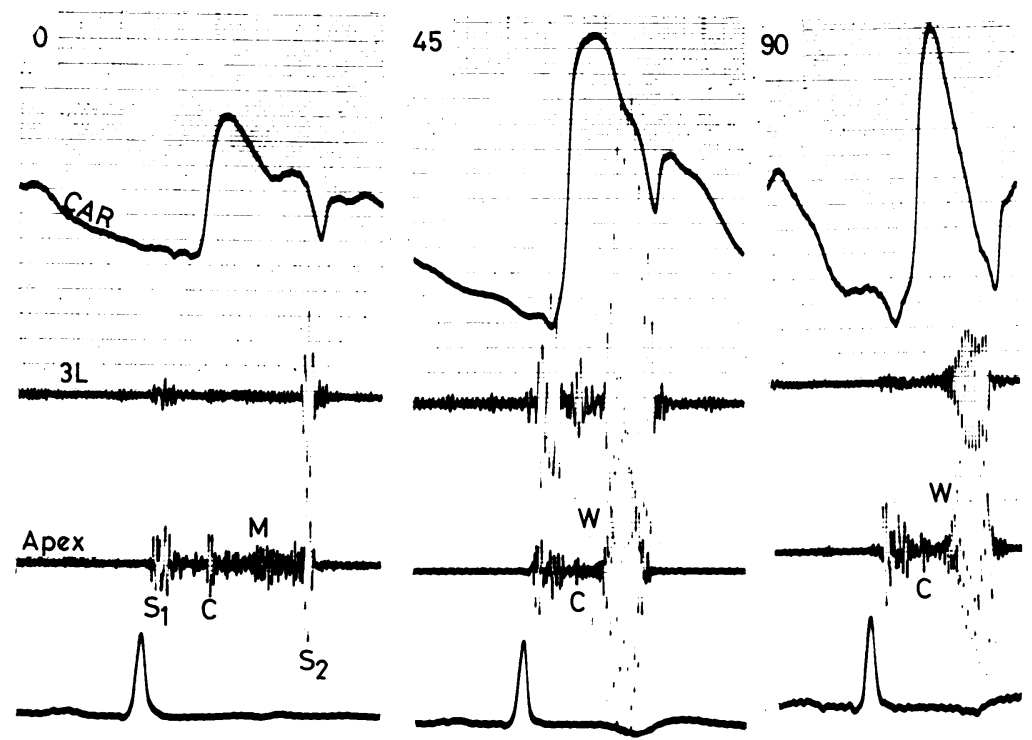

Fig. 4 Phonocardiogram from case 6 showing mid-systolic click moving progressively towards $S 1$ and diminishing in intensity with increased angle of head-up tilt. The late systolic murmur seen in supine position acquires a whooping character at steeper angles of tilt. W, whoop; other abbreviations as in previous figures.

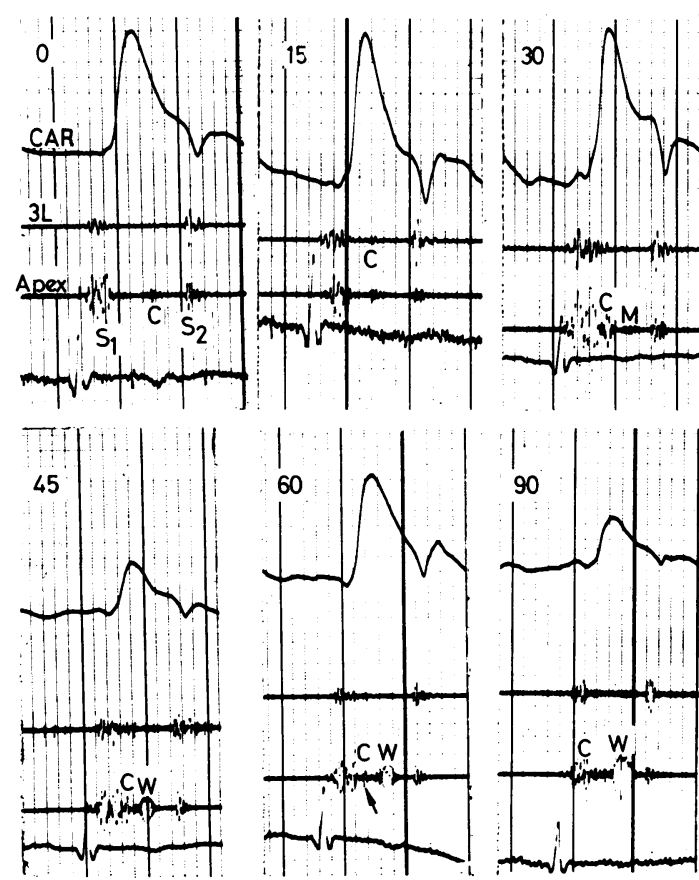

Fig. 5 Phonocardiogram from case 8 showing mid-systolic click progressively moving towards $S 1$ as the angle of head-up tilt is increased. A murmur following the click appears at $30^{\circ}$ angle and acquires a mid-systolic whooping character at steeper angle of tilt. of the angle of tilt with progressive passive headup tilt (Stafford et al., 1970), we decided to investigate the effect of the manoeuvre on the presence of a click and its timing.

In addition, the prevalence of the click syndrome among apparently healthy young adults and the theoretical possibility that severe gravitational forces encountered in military aviation might suddenly produce or seriously aggravate mitral regurgitation prompted us to study the effect of head-up tilt on the systolic murmur (Towne et al., 1971). We were particularly interested to know whether gravitational forces might result in the sudden appearance of the murmur.

We found an excellent correlation between the angle of passive head-up tilt and the decrease in the Q-C interval. Perhaps of greater interest, however, is the fact that the click was recognisable at all angles including supine in only $10(56 \%)$ of the 18 patients.

In 1 of the other 8 , the click had at a previous clinical examination been present in the supine or squatting position but absent in the sitting and standing positions. During the test the click was absent with the patient supine and during the recordings made at all five angles of tilt, but was brought out by head-down tilt after the procedure was completed. These findings suggest that the $\mathrm{CPV}$ exceeded the end-diastolic volume (EDV) in the supine position and at all angles of head-up 
tilt; this would also explain the appearance of the click with head-down tilt, a manoeuvre expected to increase EDV. In this patient, however, the click appeared intermittently soon after the adoption of $15^{\circ}$ of head-up tilt, and when present was quite loud and late systolic; these findings are not consistent with EDV less than CPV as a cause of absence of a click. During our study of the effect of atrial pacing on click position (Towne et al., 1975), we noted that while in some patients increasing the rate of pacing resulted in fusion of the click with the first sound (S1), in others the click moved toward S1 as the rate was increased but became quieter and disappeared in mid-systole. However, this phenomenon was not observed in the patient described here in whom the click was always loud and relatively late when present intermittently at $15^{\circ}$ head-up tilt. However, when progressive head-down tilt was carried out on another day (when the click was again absent supine and at all angles of head-up tilt), the click first appeared in midsystole and was initially soft and only questionably present; as the head-down tilt angle was increased, the click became later and much louder. Despite careful analysis of the data in this patient, we find it impossible to explain the presence or absence of the click in all situations by the theory of critical prolapsing volume.

Similarly, the behaviour of the click (more in terms of presence or absence than of position in systole) cannot be explained in three patients whose clicks were absent at $30^{\circ}, 45^{\circ}$, and $60^{\circ}$, at $60^{\circ}$ only, and at $30^{\circ}, 60^{\circ}$, and $90^{\circ}$, yet were present at all other angles.

On the other hand absence of the click at $90^{\circ}$ only ( 2 patients), at $45^{\circ}, 60^{\circ}$, and $90^{\circ}$ (1 patient), and at $0^{\circ}$ and $15^{\circ}$ ( 1 patient) is consistent with the critical prolapsing volume theory. In the former two instances (3 patients), EDV exceeds CPV at lesser angles of tilt so that CPV is attained during left ventricular systole resulting in a click. However, at steeper angles of tilt the reduction of EDV is such that it is exceeded by CPV and a click is not heard. In the latter instance (1 patient) CPV is less than end-systolic volume (ESV) at lower angles of tilt and since it is never achieved during systole, there can be no click. However, at the steeper angles of tilt ESV falls below CPV allowing prolapse to occur during ejection and a click to appear.

The length of the murmur of mitral regurgitation would be expected to be governed by the same factors which affect click position, while the loudness would logically relate to the same factors governing murmur intensity in any type of mitral regurgitation. The only proviso is that a factor which would normally increase murmur intensity by increasing impedance to ejection, such as alpha-adrenergic stimulation, might abolish the murmur by causing an increase in ESV such that $\mathrm{CPV}$ is never attained during systole.

Of special interest in the current study is the fact that of 16 patients without a murmur while supine, only 2 developed a murmur with progressive head-up tilt. However, also of significance is the fact that 1 of these 2 patients (the only patient who had a murmur at $0^{\circ}$ ) developed a quite loud murmur with the mild gravitational stress of $90^{\circ}$ head-up tilt.

These data are consistent with the view that once prolapse results in mitral regurgitation, further gravitational stress rapidly increases regurgitant volume. Further studies will be needed to prove or disprove this hypothesis, a matter of importance in this age of aviation when mitral valve prolapse is either increasing in incidence and prevalence, especially in young adults, or is being diagnosed more frequently (Brown et al., 1975; Markiewicz et al., 1975; Procacci et al., 1975). In addition the relative importance of increase in length as opposed to increase in intensity of the murmur must be established.

For the diagnosis of mitral valve prolapse, passive head-up tilt may be a worth-while provocative manoeuvre to reveal the presence of click or murmur. We did not attempt to assess the sensitivity or reliability of tilt in this regard, compared with the more commonly used manoeuvres (standing, squatting, amyl nitrite, neosynephrine).

The results of this study suggest that, while most of the auscultatory changes brought on by head-up tilt in subjects with mitral valve prolapse can be explained by current theories regarding the genesis of click and murmur, several patients demonstrated phenomena which suggest that other unrecognised factors may operate in determining the presence and timing of the systolic click.

\section{References}

Barlow, J. B., Bosman, C. K., Pocock, W. A., and Marchand, P. (1968). Late systolic murmurs and non-ejection ('midlate') systolic clicks. British Heart fournal, 30, 203-218.

Brown, O. R., Kloster, F. E., and DeMots, H. (1975). Incidence of mitral valve prolapse in the asymptomatic normal (abstract). Circulation, 51 and 52, Suppl. 2, 77.

Criley, J. M , Lewis, K. B., Humphries, J. O., and Ross, R. S. (1966). Prolapse of the mitral valve: clinical and cineangiocardiographic findings. British Heart fournal, 28, 488-496.

Dillon, J. C., Haine, C. L., Chang, S., and Feigenbaum, H. (1971). Use of echocardiography in patients with prolapsed mitral valve. Circulation, 43, 503-507.

Fontana, M. E., Wooley, C. F., Leighton, R. F., and Lewis, R. P. (1975). Postural changes in left ventricular and mitral valvular dynamics in the systolic click-late systolic murmur syndrome. Circulation, 51, 165-173. 
Jeresaty, R. M. (1973). Mitral valve prolapse-click syndrome. Progress in Cardiovascular Diseases, 15, 623-652.

Kerber, R. E., Isaeff, D. M., and Hancock, E. W. (1971). Echocardiographic patterns in patients with the syndrome of systolic click and late systolic murmur. New England fournal of Medicine, 284, 691-693.

Markiewicz, W., Stoner, J., London, E., Hunt, S. A., and Popp, R. L. (1975). Mitral valve prolapse in one hundred presumably healthy females (abstract). Circulation, 51 and 52, Suppl. 2, 77.

Mathey, D. G., Decoodt, P. R., Allen, H. N., and Swan, H. J. C. (1976). The determinants of onset of mitral valve prolapse in the systolic click-late systolic murmur syndrome. Circulation, 53, 872-878.

Popp, R. L., Brown, O. R., Silverman, J. F., and Harrison, D. C. (1974). Echocardiographic abnormalities in the mitral valve prolapse syndrome. Circulation, 49, 428-433.

Procacci, P. M., Savran, S. V., Schreiter, S. L., and Bryson, A. L. (1975). Clinical frequency and implications of mitral valve prolapse in the female population (abstract). Circulation, 51 and 52, Suppl. 2, 78.

Ranganathan, N., Silver, M. D., Robinson, R. I., Kostuk, W. J., Felderhof, C, H., Patt, N. L., Wilson, J. K., and Wigle, E. D. (1973). Angiographic-morphologic correlation in patients with severe mitral regurgitation due to prolapse of the posterior mitral valve leaflet. Circulation, 48, 514-518.

Stafford, R. W., Harris, W. S., and Weissler, A. M. (1970). Left ventricular systolic time intervals as indices of postural circulatory stress in man. Circulation, 41, 485-492.

Towne, W. D., Rahimtoola, S. H., Rosen, K. M., Casten, C. P., and Gunnar, R. M. (1971). Systolic prolapse of the mitral valve: possible aeromedical significance. Aerospace Medicine, 42, 341-344.

Towne, W. D., Rahimtoola, S. H., Sinno, M. Z., Loeb, H. S., Rosen, K. M., and Gunnar, R. M. (1975). The effects of right atrial and ventricular pacing on the auscultatory findings in patients with mitral valve prolapse. Circulation, 51, 988-996.

Winkle, R. A., Goodman, D. J., and Popp, R. L. (1975). Simultaneous echocardiographic-phonocardiographic recordings at rest and during amyl nitrite administration in patients with mitral valve prolapse. Circulation, 51, 522-529.

Requests for reprints to $\mathrm{Dr}$ William D. Towne, Division of Adult Cardiology, Cook County Hospital, 1825 W. Harrison Street, Chicago, Illinois 60612, USA. 